\title{
Use of salivary liver function markers for the prognosis of deaddiction in alcohol dependent non-smokers and smokers: A before and after study
}

\author{
JB Honnamurthy ${ }^{1, *}$, Shivashankara $\mathrm{AR}^{2}$, Avinash $\mathrm{SS}^{3}$, John P Mathai ${ }^{4}$, Malathi $\mathrm{M}^{5}$ \\ ${ }^{1}$ Research Scholar, ${ }^{2,3}$ Associate Professor, ${ }^{4,5}$ Professor, ${ }^{\mathbf{1 , 2 , 3 , 5}}$ Dept. of Biochemistry, ${ }^{4}$ Dept. of Psychiatry, Father \\ Muller Medical College, Mangalore, India
}

*Corresponding Author:

Email: honnu2012@gmail.com

\begin{abstract}
Alcohol deaddiction improves the liver function markers in blood as well as saliva in both smokers and nonsmokers. The study was conducted to observe the changes in liver function markers after deaddiction in both blood as well as saliva. The study included 83 alcohol dependent subjects, among whom 45 were smokers and 38 were non-smokers. They were followed up after deaddiction for 19-21 days. The liver function markers GGT- $\gamma$ glutamyl transferase, AST- Aspartate transaminase, ALT- Alanine transaminase, TP- Total protein, and AlbAlbumin were measured spectrophotometrically before and after deaddiction in saliva as well as blood. Paired ' $t$ ' test was done to analyse the significance of their changes in before and after deaddiction. There was no difference in NR (normalization rate) of GGT, AST and ALT between alcohol dependent smokers and non-smokers in saliva as well as blood. NR of TP and Alb was better in non-smokers compared to smokers. GGT ( $\mathrm{p}<.001)$, AST $(\mathrm{p}<.001)$ and ALT $(\mathrm{p}<.001)$ have decreased and SFR has increased significantly in all groups after withdrawal. Elevation after withdrawal of $\mathrm{TP}$ and Alb were not significant. Elevation of Alb after withdrawal was not significant. In both alcohol dependent smokers as well as non-smokers, salivary GGT ( $<<.001)$, AST ( $<<.001)$ and ALT (p<.001) levels can be used instead of blood levels to monitor the prognosis of deaddiction, but TP and Alb cannot be used for the same.
\end{abstract}

Keywords: Aminotransferases, Alcohol withdrawal, Alcohol dependence, $\gamma$-glutamyl transferase.

Received: $13^{\text {th }}$ October, 2017

\section{Introduction}

Alcohol is a psychoactive substance which may cause dependence. The World Health Organization (WHO) recently stated that harmful use of alcohol ranks among the top five risk factors for disease, disability and death throughout the world. Globally, alcohol abuse causes approximately 3.3 million deaths every year or $5.9 \%$ of all deaths, and $5.1 \%$ of the global burden of disease is attributable to alcohol consumption. ${ }^{1}$

Alcohol dependents are addicted to smoking which may increase the pathogenic effects on the liver. ${ }^{2}$ The smoking and alcohol dependence has a common genetic vulnerability. ${ }^{3}$

Alcohol biomarkers play a significant role in the early diagnosis of alcohol abuse, alcoholrelated organ damages, and assessment of alcoholism therapy. The liver function markers assays are used in the detection of excessive alcohol consumption, acute ethanol intoxication and in monitoring of alcohol consumption. Alcohol biomarkers can demonstrate the effects of alcohol on the body, classified as indirect markers, or as direct markers. The traditional alcohol biomarkers are alanine aminotransferase (ALT), aspartate aminotransferase (AST), and
Gamma glutamyl transferase (GGT). The activities of GGT, AST and ALT are elevated in chronic alcoholics ${ }^{4}$ and indicate generalized hepatic damage. ${ }^{5}$

Saliva like blood contains wide range of enzymes and proteins as biological markers. Blood collection is intrusive however salivary collection is non-invasive and economical. ${ }^{6}$ The constituents of the blood enter the saliva by mechanism such as active transport, passive diffusion, leakage or ultrafiltration. ${ }^{7}$ Saliva can be used as a substitute for blood in the diagnosis and prognosis of the disease. ${ }^{8}$

Our study hypothesis is that alcohol deaddiction improves the liver function markers in blood as well as saliva in both smokers and non-smokers. The studies to prove the effect of smoking on the alcohol induced changes after deaddiction in liver function enzymes in both blood as well as saliva are rare. Hence the study was conducted to observe the changes in liver function markers after deaddiction in both blood as well as saliva. We also intend to find if there is any difference in normalization of the liver function markers between alcohol dependent smokers and non-smokers. 


\section{Materials and Methods}

The study is a prospective cohort study, conducted at Vailankanni deaddiction centre, Father Muller Medical College, Mangalore, for a period of six months from Jan 2016 to July 2016. The patients admitted for deaddiction were diagnosed as alcohol dependence by a treating psychiatrist based on The ICD-10 (the $10^{\text {th }}$ revision of the international statistical classification of diseases) classification of mental and behavioural disorders: diagnostic criteria for research WHO. ${ }^{9}$ All the subjects were followed up for 21 days after deaddiction for the outcome of normalization in liver function markers. Of the 150 alcohol dependent participants recruited for the study, 83 subjects were found eligible and agreed to participate voluntarily, by providing information about the duration and amount of alcohol consumption, smoking and family history. Based on the history of smoking we categorised alcohol dependents into alcohol dependent smokers and non-smokers. Among them 45 subjects were alcohol dependent smokers with age $36 \pm 10$ years (mean \pm SD) ranged from 21-60 years. And 38 subjects were alcohol dependent non-smokers of age $39 \pm 10$ years $($ mean $\pm \mathrm{SD})$ ranged from 23-65 years. The recruited participants with the following potential confounders as in systemic illness such as cancer, thyroid disorder, alcohol liver disease, inflammatory disease, psychiatric illness, abstinence from alcohol prior to admission, and substance other than alcohol and nicotine dependence were excluded from the study. The written informed was consent was taken from the study subjects. This study was approved by Father Muller Institutional Ethics committee.

For collection of unstimulated whole saliva, subjects were refrained from taking food for 1 hour before collection and advised to clean the mouth by rinsing with water to remove any food debris. The saliva was collected between 9AM$11 \mathrm{AM}$ into a sterile containers avoiding forcible spitting as specified by the method of Navzesh. ${ }^{10}$
The time and amount of saliva collected is recorded for the calculation of salivary flow rate (SFR). The saliva samples were centrifuged at $3000 \mathrm{rpm}$ for 15 minutes and the supernatant was collected. Blood was collected after the saliva collection in EDTA or plain vaccutainers taking aseptic precautions, serum is separated by centrifugation. The saliva and serum samples collected were stored in $-80^{\circ} \mathrm{C}$ until the assay done.

Biochemical assays for saliva and serum GGT, AST, ALT, TP Alb, and Glb (globulin) were performed in all subjects. GGT activity was assayed by carboxy substrate method. ${ }^{11}$ Amino transferases activity both $\mathrm{AST}^{12}$ and $\mathrm{ALT}^{13}$ were assayed by modified IFCC method. Albumin was assayed by Bromocresol green dye binding method. ${ }^{14}$ The salivary TP was assayed by Lowry's method ${ }^{15}$ and serum TP was assayed by Biuret's reagent method. ${ }^{16} \mathrm{Glb}$ was calculated by TP-Alb. Paired ' $t$ ' test was employed to assess the changes in salivary and blood liver function markers before and after withdrawal. Paired correlation was done to assess the strength of association between before and after withdrawal levels of liver function markers. SPSS version 24 software was used for the analysis. $p$ value $<.05$ was considered statistically significant.

\section{Results}

At the time of study the subjects recruited was 83(45 alcohol dependent smokers and 38 alcohol dependent non-smokers) there was no loss to follow up. The descriptive data is shown in Table 1. Results of paired' $t$ ' test of salivary and blood liver function markers before and after withdrawal in alcohol dependent smokers and non-smokers are shown in Table 2 and 3 respectively.

The correlation of paired samples of salivary and blood liver function markers in alcohol dependents, smokers and non-smokers are shown in Table 4 and 5 respectively.

Table 1: Demographic variables of the study population

\begin{tabular}{|l|c|}
\hline Demographic variables & $\begin{array}{c}\text { Alcohol dependents (both smokers } \\
\text { + non-smokers) (n-83) }\end{array}$ \\
\hline Age in years $\{$ Mean \pm SD $($ range $)\}$ & $37.5 \pm 10(21-65)$ \\
\hline $\begin{array}{l}\text { Duration of alcohol consumption in } \\
\text { years Median (IQR) }\end{array}$ & $15(8)$ \\
\hline $\begin{array}{l}\text { Amount of alcohol consumption in } \mathrm{ml} \\
\text { per day }\end{array}$ & $\begin{array}{c}<180 \mathrm{ml}-\mathrm{n}=18,180-360 \mathrm{ml}-\mathrm{n}=37, \\
360-540 \mathrm{ml}-\mathrm{n}=20,>540 \mathrm{ml}-\mathrm{n}=8 .\end{array}$ \\
\hline Family history of Alcohol dependence & Present $-36(43 \%)$, Absent- $47(56 \%)$ \\
\hline History of smoking present & $\mathrm{n}-45$ \\
\hline History of smoking absent & 6.38 \\
\hline Daily consumption in years & 3.75 \\
\hline Morning consumption in years & 6.61 \\
\hline
\end{tabular}


Table 2: Paired' $t$ ' test of salivary liver function markers before and after withdrawal in alcohol dependent smokers and non-smokers

\begin{tabular}{|c|c|c|c|c|c|c|c|}
\hline $\begin{array}{c}\text { Salivary } \\
\text { Liver } \\
\text { function } \\
\text { markers }\end{array}$ & $\begin{array}{c}\text { GGT } \\
\text { (IU/L) } \\
\text { Mean } \\
\text { difference } \\
\pm \text { SE } \\
(95 \% \text { CI }) \\
\end{array}$ & $\begin{array}{c}\text { AST }(\text { IU/L) } \\
\text { Mean } \\
\text { difference } \\
\pm \text { SE } \\
(95 \% \mathrm{CI})\end{array}$ & $\begin{array}{c}\text { ALT } \\
\text { (IU/L) } \\
\text { Mean } \\
\text { difference } \\
\pm \text { SE } \\
(95 \% \mathrm{CI}) \\
\end{array}$ & $\begin{array}{c}\text { TP }(g / d l) \\
\text { Mean } \\
\text { difference } \\
\pm \text { SE } \\
(95 \% \mathrm{CI})\end{array}$ & $\begin{array}{c}\text { Alb }(g / d l) \\
\text { Mean } \\
\text { difference } \\
\pm \text { SE } \\
(95 \% \mathrm{CI})\end{array}$ & $\begin{array}{c}\text { SFR }(\mathrm{ml} / \mathrm{min}) \\
\text { Mean } \\
\text { difference } \\
\pm \mathrm{SE} \\
(95 \% \mathrm{CI})\end{array}$ & $\begin{array}{c}\text { Glb } \\
\text { (g/dl) } \\
\text { Mean } \\
\text { difference } \\
\pm \text { SE } \\
(95 \% \mathrm{CI}) \\
\end{array}$ \\
\hline \multirow[t]{2}{*}{$\begin{array}{l}\text { Alcohol } \\
\text { dependents }\end{array}$} & $\begin{array}{c}4.32 \pm .37 \\
(3.5-5)\end{array}$ & $\begin{array}{c}6.07 \pm .52 \\
(5.04-7.1)\end{array}$ & $\begin{array}{c}3.51 \pm .4 \\
(2.7-4.3)\end{array}$ & $\begin{array}{c}.004 \pm .009 \\
(-.02-.01)\end{array}$ & $\begin{array}{l}-.01 \pm .005 \\
(-.02-.00)\end{array}$ & $\begin{array}{c}.05 \pm .01 \\
(-.08--.03)\end{array}$ & $\begin{array}{l}.001 \pm .001 \\
(-.01-.03)\end{array}$ \\
\hline & $\begin{array}{c}* \mathrm{p}-<.001 \\
\mathrm{NR}-48.3 \%\end{array}$ & $\begin{array}{c}* \mathrm{p}-<.001 \\
\text { NR-48.8\%\% }\end{array}$ & $\begin{array}{c}* \mathrm{p}-<.001 \\
\mathrm{NR}-43.4 \%\end{array}$ & $\begin{array}{c}\mathrm{p}-.59 \\
\text { NR-(- } 3.38 \%)\end{array}$ & $\begin{array}{c}\mathrm{p}-.05 \\
\text { NR-(-28.1\%) }\end{array}$ & $\begin{array}{c}* \mathrm{p}-<.001 \\
\mathrm{NR}-(-18.9 \%)\end{array}$ & $\begin{array}{c}\mathrm{p}-.511 \\
\text { NR-(-6.3\%) }\end{array}$ \\
\hline \multirow{2}{*}{$\begin{array}{l}\text { Alcohol } \\
\text { dependent } \\
\text { smokers }\end{array}$} & $\begin{array}{c}4.08 \pm .5 \\
(3.06-5.1) \\
\end{array}$ & $\begin{array}{c}6.5 \pm .79 \\
(4.9-8.1) \\
\end{array}$ & $\begin{array}{l}3.19 \pm .54 \\
(2.1-4.2) \\
\end{array}$ & $\begin{array}{c}.001 \pm .01 \\
(-.026-.028) \\
\end{array}$ & $\begin{array}{l}-.001 \pm .003 \\
(-.008-.005) \\
\end{array}$ & $\begin{array}{c}-.05 \pm .01 \\
(-.08--.02) \\
\end{array}$ & $\begin{array}{c}.003 \pm .01 \\
(-.02-.03) \\
\end{array}$ \\
\hline & $\begin{array}{c}* \mathrm{p}-<.001 \\
\mathrm{NR}-48.1 \%\end{array}$ & $\begin{array}{c}* \mathrm{p}-<.001 \\
\mathrm{NR}-49.2 \% \\
\end{array}$ & $\begin{array}{l}* \mathrm{p}-<.001 \\
\mathrm{NR}-41 \%\end{array}$ & $\begin{array}{c}\mathrm{p}-.93 \\
\text { NR- } 0.72 \% \\
\end{array}$ & $\begin{array}{c}\mathrm{p}-.64 \\
\text { NR-(-3.8\%) }\end{array}$ & $\begin{array}{c}* \mathrm{p}-.001 \\
\text { NR-(-18.3\%) }\end{array}$ & $\begin{array}{c}\mathrm{p}-.844 \\
\text { NR-(-2.3\%) } \\
\end{array}$ \\
\hline \multirow{2}{*}{$\begin{array}{l}\text { Alcohol } \\
\text { dependent } \\
\text { non- } \\
\text { smokers }\end{array}$} & $\begin{array}{c}4.6 \pm .55 \\
(3.4-5.7)\end{array}$ & $\begin{array}{c}5.5 \pm .63 \\
(4.2-6.8)\end{array}$ & $\begin{array}{c}3.8 \pm .61 \\
(2.6-5.1)\end{array}$ & $\begin{array}{l}-.01 \pm .01 \\
(-.03-.01)\end{array}$ & $\begin{array}{c}-.02 \pm .01 \\
(-.04-.0006)\end{array}$ & $\begin{array}{c}-.06 \pm .02 \\
(-.10-(-.02))\end{array}$ & $\begin{array}{c}.01 \pm .01 \\
(-.02-.04)\end{array}$ \\
\hline & $\begin{array}{c}* \mathrm{p}-<.001 \\
\mathrm{NR}-48.4 \%\end{array}$ & $\begin{array}{c}* \mathrm{p}-<.001 \\
\text { NR-48.08\% }\end{array}$ & $\begin{array}{c}* \mathrm{p}-<.001 \\
\mathrm{NR}-45.8 \%\end{array}$ & $\begin{array}{c}\mathrm{p}-.31 \\
\text { NR- }(-8.84 \%)\end{array}$ & $\begin{array}{c}\mathrm{p}-.05 \\
\text { NR-(-54.3\%) }\end{array}$ & $\begin{array}{c}* \mathrm{p}-.004 \\
\text { NR-(-19.5\%) }\end{array}$ & $\begin{array}{c}\mathrm{p}-.466 \\
\text { NR- }(-12 \%)\end{array}$ \\
\hline
\end{tabular}

Legend to Table 2: GGT- $\gamma$ glutamyl transferase, AST- Aspartate transaminase, ALT- Alanine transaminase, TP- Total protein, Alb-Albumin, SFR- Salivary flow rate, SE- Standard error, CIConfidence interval, NR- Normalization rate, $\mathrm{p}<.05$ is statistically significant. NR $=$ (Levels before withdrawal-Level after withdrawal) X100/Level before withdrawal. There was no difference in NR of GGT, AST ALT and SFR between the groups. NR of TP and Alb was better in non-smokers compared to smokers. GGT, AST and ALT have decreased and SFR has increased significantly in all groups after withdrawal. Elevation after withdrawal of $\mathrm{TP}$, Alb and Glb were not significant. p<.05 significant.

Table 3: Paired' $t$ ' test of Blood liver function markers before and after withdrawal in alcohol dependent smokers and non-smokers

\begin{tabular}{|c|c|c|c|c|c|c|}
\hline $\begin{array}{c}\text { Blood } \\
\text { Liver } \\
\text { function } \\
\text { markers }\end{array}$ & $\begin{array}{c}\text { GGT }(\text { IU/L) } \\
\text { Mean } \\
\text { difference } \\
\pm \text { SE }(95 \% \mathrm{CI})\end{array}$ & $\begin{array}{c}\text { AST }(\text { IU/L) } \\
\text { Mean } \\
\text { difference } \\
\pm \text { SE }(95 \% \mathrm{CI})\end{array}$ & $\begin{array}{c}\text { ALT (IU/L) } \\
\text { Mean } \\
\text { difference } \\
\pm \text { SE }(95 \% \mathrm{CI})\end{array}$ & $\begin{array}{c}\mathrm{TP}(\mathrm{g} / \mathrm{dl}) \\
\text { Mean } \\
\text { difference } \\
\pm \mathrm{SE}(95 \% \mathrm{CI})\end{array}$ & $\begin{array}{c}\text { Alb }(\mathrm{g} / \mathrm{dl}) \\
\text { Mean } \\
\text { difference } \\
\pm \mathrm{SE}(95 \% \mathrm{CI})\end{array}$ & $\begin{array}{c}\text { Glb } \\
\text { (g/dl) Mean } \\
\text { difference } \\
\pm \mathrm{SE}(95 \% \mathrm{CI})\end{array}$ \\
\hline \multirow[t]{2}{*}{$\begin{array}{l}\text { Alcohol } \\
\text { dependents }\end{array}$} & $\begin{array}{c}214.8 \pm 33.3 \\
(148-281)\end{array}$ & $\begin{array}{c}70.3 \pm 7.5 \\
(55.4-85)\end{array}$ & $\begin{array}{c}43.6 \pm 5 \\
(33.6-53.7)\end{array}$ & $\begin{array}{c}-.2 \pm .06 \\
(-.34-(-.06))\end{array}$ & $\begin{array}{l}-.04 \pm .05 \\
(-.15-.05)\end{array}$ & $\begin{array}{c}-.2 \pm .05 \\
(-.3-(-.05))\end{array}$ \\
\hline & $\begin{array}{c}* \mathrm{p}-<.001 \\
\text { NR- } 66.7 \%\end{array}$ & $\begin{array}{c}* \mathrm{p}-<.001 \\
\text { NR- } 61.83 \%\end{array}$ & $\begin{array}{c}{ }^{*} \mathrm{p}-<.001 \\
\mathrm{NR}-55.7 \%\end{array}$ & $\begin{array}{c}* \mathrm{p}-.004 \\
\text { NR- }(-2.7 \%)\end{array}$ & $\begin{array}{c}\mathrm{p}-.352 \\
\text { NR-(-1.1\%) }\end{array}$ & $\begin{array}{c}* \mathrm{p}-.005 \\
\text { NR- }(-5.3 \%)\end{array}$ \\
\hline \multirow{2}{*}{$\begin{array}{l}\text { Alcohol } \\
\text { dependent } \\
\text { smokers }\end{array}$} & $\begin{array}{c}264 \pm 54 \\
(153-374)\end{array}$ & $\begin{array}{c}70.9 \pm 11.4 \\
(47.9-94)\end{array}$ & $\begin{array}{c}41.3 \pm 6.8 \\
(27-55)\end{array}$ & $\begin{array}{l}-.14 \pm .09 \\
(-.33-.04)\end{array}$ & $\begin{array}{l}-.02 \pm .07 \\
(-.17-.1)\end{array}$ & $\begin{array}{l}-.012 \pm .06 \\
(-.3-.01)\end{array}$ \\
\hline & $\begin{array}{c}* \mathrm{p}-<.001 \\
\text { NR- } 70.5 \%\end{array}$ & $\begin{array}{c}* \mathrm{p}-<.001 \\
\text { NR- } 60.1 \%\end{array}$ & $\begin{array}{c}* \mathrm{p}-<.001 \\
\text { NR- } 52.1 \%\end{array}$ & $\begin{array}{c}\mathrm{p}-.14 \\
\text { NR- }(-1.9 \%)\end{array}$ & $\begin{array}{c}\mathrm{p}-.69 \\
\text { NR- }(-0.7 \%)\end{array}$ & $\begin{array}{c}\text { p-.078 } \\
\text { NR- }(-3.9 \%)\end{array}$ \\
\hline \multirow{2}{*}{$\begin{array}{l}\text { Alcohol } \\
\text { dependent } \\
\text { non- } \\
\text { smokers }\end{array}$} & $\begin{array}{c}156 \pm 31.9 \\
(91.8-221)\end{array}$ & $\begin{array}{c}69.6 \pm 9.4 \\
(50.4-88.7)\end{array}$ & $\begin{array}{c}46.3 \pm 7.6 \\
(30.9-61.8)\end{array}$ & $\begin{array}{c}-.27 \pm .09 \\
(-.47(-.07))\end{array}$ & $\begin{array}{l}-.07 \pm .07 \\
(-.22-.07)\end{array}$ & $\begin{array}{c}-.2 \pm .09 \\
(-.4-(-0.02))\end{array}$ \\
\hline & $\begin{array}{c}* \mathrm{p}-<.001 \\
\text { NR- } 59.9 \%\end{array}$ & $\begin{array}{l}* \mathrm{p}-<.001 \\
\mathrm{NR}-63 \%\end{array}$ & $\begin{array}{c}* \mathrm{p}-<.001 \\
\text { NR- } 60 \%\end{array}$ & $\begin{array}{c}\text { *p-.007 } \\
\text { NR- }(-3.7 \%)\end{array}$ & $\begin{array}{c}\mathrm{p}-.345 \\
\text { NR-(-1.6\%) }\end{array}$ & $\begin{array}{c}* \mathrm{p}-.03 \\
\text { NR- }(-6.9 \%)\end{array}$ \\
\hline
\end{tabular}

Legend to table No.3: GGT- $\gamma$ glutamyl trasnferase, AST- Aspartate transaminase, ALT- Alanine transaminase, TP- Total protein, Alb-Albumin, SFR- Salivary flow rate, SE- Standard error, CIConfidence interval, NR- Normalization rate, $\mathrm{p}<.05$ is statistically significant. NR $=($ Levels before withdrawal-Level after withdrawal) X100/Level before withdrawal. There was no difference in NR of GGT, AST and ALT between the groups. NR of TP and Alb was better in non smokers compared to smokers. GGT, AST and ALT have decreased significantly in all groups after withdrawal. TP elevated significantly after withdrawal in non-smokers. Elevation of Alb after withdrawal was not significant. Glb was significantly decreased in smokers after withdrawal. $\mathrm{p}<.05-$ significant.

Table 4: Paired samples Correlation of salivary liver function markers in alcohol dependents, smokers and non-smokers

\begin{tabular}{|l|c|c|c|c|c|c|c|c|c|}
\hline & \multicolumn{3}{|c|}{ Alchol dependent } & \multicolumn{2}{c|}{ Alcohol dependent smokers } & \multicolumn{3}{c|}{ Alcohol dependent non-smoker } \\
\hline & Mean \pm & Mean \pm & r value & Mean \pm & Mean \pm & r value & Mean \pm & Mean \pm & r value \\
& SE & SE & P value & SE & SE & PW value & SE & SE & $\begin{array}{c}\text { AW } \\
\text { P value }\end{array}$ \\
& BW & AW & & & AW & & \\
\hline GGT & $8.9 \pm$ & $4.65 \pm$ & 0.792 & $8.5 \pm$ & $4.4 \pm$ & 0.77 & $9.56 \pm$ & $4.96 \pm$ & 0.81 \\
(IU/L) & 0.57 & 0.33 & $* \mathrm{p}<.001$ & 0.78 & 0.45 & $* \mathrm{p}<.001$ & 0.87 & 0.49 & $* \mathrm{p}<.001$ \\
\hline AST & $12.45 \pm$ & $6.37 \pm$ & 0.833 & $13.24 \pm$ & $6.7 \pm$ & $0.8 * \mathrm{p}<.001$ & $11.5 \pm$ & $5.97 \pm$ & 0.88 \\
\hline
\end{tabular}

International Journal of Clinical Biochemistry and Research, January-March, 2018;5(1):35-40 37 


\begin{tabular}{|l|c|c|c|c|c|c|c|c|c|}
\hline (IU/L) & 0.9 & 0.59 & $* \mathrm{p}<.001$ & 1.25 & 0.72 & & 1.3 & 0.99 & $* \mathrm{p}<.001$ \\
\hline ALT & $8.18 \pm$ & $4.6 \pm$ & 0.843 & $7.84 \pm$ & $4.64 \pm$ & 0.78 & $8.6 \pm$ & $4.7 \pm$ & 0.9 \\
(IU/L) & 0.65 & 0.34 & $* \mathrm{p}<.001$ & 0.82 & 0.46 & $* \mathrm{p}<.001$ & 1.04 & 0.52 & $* \mathrm{p}<.001$ \\
\hline TP & $0.14 \pm$ & $0.15 \pm$ & 0.323 & $0.153 \pm$ & $0.152 \pm$ & 0.32 & $0.14 \pm$ & $0.15 \pm$ & 0.32 \\
$\mathbf{( g / d l})$ & 0.008 & 0.006 & $* \mathrm{p}=.003$ & 0.013 & 0.009. & $* \mathrm{p}<.029$ & 0.01 & 0.01 & $* \mathrm{p}=.048$ \\
\hline $\mathbf{A l b}$ & $0.04 \pm$ & $0.05 \pm$ & 0.376 & $0.039 \pm$ & $0.041 \pm$ & 0.74 & $0.04 \pm$ & $0.06 \pm$ & $0.26 \mathrm{p}=.11$ \\
$\mathbf{( g / d l})$ & 0.003 & 0.006 & $* \mathrm{p}<.001$ & 0.005 & 0.004 & $* \mathrm{p}<.001$ & 0.005 & 0.01 & 0.27 \\
\hline $\mathbf{G l b}$ & $0.1 \pm$ & $0.09 \pm$ & 0.327 & $0.113 \pm$ & $0.11 \pm$ & 0.376 & $0.09 \pm$ & $0.08 \pm$ & 0.091 \\
$\mathbf{( g / d l})$ & 0.009 & 0.008 & $* \mathrm{p}=.003$ & 0.014 & 0.009 & $* \mathrm{p}=.011$ & 0.01 & 0.014 & $\mathrm{p}=.097$ \\
\hline $\mathbf{S F R}$ & $0.30 \pm$ & $0.36 \pm$ & 0.758 & $0.29 \pm$ & $0.35 \pm$ & 0.83 & $0.32 \pm$ & $0.38 \pm$ & 0.68 \\
$\mathbf{( m l / m i n})$ & 0.018 & 0.014 & $* \mathrm{p}<.001$ & 0.026 & 0.019 & $* \mathrm{p}<.001$ & 0.03 & 0.02 & $* \mathrm{p}<.001$ \\
\hline
\end{tabular}

Legend to Table 4: GGT- $\gamma$ glutamyl trasnferase, AST- Aspartate transaminase, ALT- Alanine transaminase, TP- Total protein, Alb-Albumin, SFR- Salivary flow rate, SE- Standard error. BWBefore withdrawal, AW- After withdrawal, r-correlation coefficient. All the parameters show significant correlation between before and after withdrawal levels except TP, Alb and Glb in alcohol dependent non-smokers. $\mathrm{p}<.05$ - significant.

Table 5: Paired samples Correlation of blood liver function markers in alcohol dependents, smokers and non-smokers

\begin{tabular}{|c|c|c|c|c|c|c|c|c|c|}
\hline & \multicolumn{3}{|c|}{ Alcohol dependent } & \multicolumn{3}{|c|}{ Alcohol dependent smokers } & \multicolumn{3}{|c|}{ Alcohol dependent non-smoker } \\
\hline & $\begin{array}{c}\text { Mean } \pm \\
\text { SE } \\
\text { BW }\end{array}$ & $\begin{array}{c}\text { Mean } \pm \\
\text { SE } \\
\text { AW }\end{array}$ & $\begin{array}{l}r \text { value } \\
p \text { value }\end{array}$ & $\begin{array}{c}\text { Mean } \pm \\
\text { SE } \\
\text { BW }\end{array}$ & $\begin{array}{c}\text { Mean } \pm \\
\text { SE } \\
\text { AW }\end{array}$ & $\begin{array}{l}r \text { value } \\
p \text { value }\end{array}$ & $\begin{array}{c}\text { Mean } \pm \\
\text { SE } \\
\text { BW }\end{array}$ & $\begin{array}{c}\text { Mean } \pm \\
\text { SE } \\
\text { AW }\end{array}$ & $\begin{array}{l}r \text { value } \\
p \text { value }\end{array}$ \\
\hline GGT (IU/L) & $\begin{array}{c}322 \pm \\
41 \\
\end{array}$ & $\begin{array}{c}107 \pm \\
11 \\
\end{array}$ & $\begin{array}{c}0.772 \\
* \mathrm{p}<.001\end{array}$ & $\begin{array}{c}374.4 \pm \\
64 \\
\end{array}$ & $\begin{array}{c}110.3 \pm \\
14.8\end{array}$ & $\begin{array}{c}0.697 \\
* \mathrm{p}<.001 \\
\end{array}$ & $\begin{array}{c}261.4 \pm \\
47.6\end{array}$ & $\begin{array}{c}104.9 \pm \\
16.8 \\
\end{array}$ & $\begin{array}{c}0.95 \\
* \mathrm{p}<.001\end{array}$ \\
\hline $\begin{array}{l}\text { AST } \\
\text { (IU/L) }\end{array}$ & $\begin{array}{c}113 \pm \\
9.4\end{array}$ & $\begin{array}{c}43.4 \pm \\
3.2\end{array}$ & $\begin{array}{c}0.710 \\
* \mathrm{p}<.001\end{array}$ & $\begin{array}{c}116.6 \pm \\
14.4\end{array}$ & $\begin{array}{c}45.6 \pm \\
5.1\end{array}$ & $\begin{array}{c}0.686 \\
* \mathrm{p}<.001\end{array}$ & $\begin{array}{c}110.5 \pm \\
12.1\end{array}$ & $\begin{array}{c}40.9 \pm \\
4\end{array}$ & $\begin{array}{c}0.761 \\
* \mathrm{p}<.001\end{array}$ \\
\hline $\begin{array}{l}\text { ALT } \\
\text { (IU/L) }\end{array}$ & $\begin{array}{c}78.4 \pm \\
6.9\end{array}$ & $\begin{array}{c}34.7 \pm \\
2.9\end{array}$ & $\begin{array}{c}0.766 \\
* \mathrm{p}<.001\end{array}$ & $\begin{array}{c}79.4 \pm \\
9\end{array}$ & $\begin{array}{c}38.1 \pm \\
4\end{array}$ & $\begin{array}{c}0.712 \\
p<.001\end{array}$ & $\begin{array}{c}77.3 \pm \\
10.8\end{array}$ & $\begin{array}{c}30.1 \pm \\
4.2\end{array}$ & $\begin{array}{c}0.837 \\
\mathrm{p}<.001\end{array}$ \\
\hline $\begin{array}{l}\text { TP } \\
\text { (g/dl) }\end{array}$ & $\begin{array}{l}7.4 \pm \\
0.05\end{array}$ & $\begin{array}{l}7.6 \pm \\
0.05\end{array}$ & $\begin{array}{c}0.274 \\
* \mathrm{p}=.012\end{array}$ & $\begin{array}{l}7.4 \pm \\
0.08\end{array}$ & $\begin{array}{l}7.5 \pm \\
0.07\end{array}$ & $\begin{array}{l}0.179 \\
\mathrm{p}=.24\end{array}$ & $\begin{array}{l}7.4 \pm \\
0.09\end{array}$ & $\begin{array}{l}7.7 \pm \\
0.08\end{array}$ & $\begin{array}{c}0.376 \\
* \mathrm{p}=.02\end{array}$ \\
\hline $\begin{array}{l}\text { Alb } \\
\text { (g/dl) }\end{array}$ & $\begin{array}{c}4.42 \pm \\
0.04\end{array}$ & $\begin{array}{c}4.47 \pm \\
0.03\end{array}$ & $\begin{array}{c}0.105 \\
\mathrm{p}=.343\end{array}$ & $\begin{array}{l}4.4 \pm \\
0.05 \\
\end{array}$ & $\begin{array}{l}4.5 \pm \\
0.05\end{array}$ & $\begin{array}{c}0.05 \\
p=.74\end{array}$ & $\begin{array}{l}4.4 \pm \\
0.06 \\
\end{array}$ & $\begin{array}{l}4.5 \pm \\
0.05\end{array}$ & $\begin{array}{c}0.172 \\
p=.303\end{array}$ \\
\hline $\begin{array}{l}\text { Glb } \\
\text { (g/dl) }\end{array}$ & $\begin{array}{c}2.98 \pm \\
0.05\end{array}$ & $\begin{array}{l}3.1 \pm \\
0.05\end{array}$ & $\begin{array}{c}0.475 \\
* \mathrm{p}<.001 \\
\end{array}$ & $\begin{array}{c}2.96 \pm \\
0.07\end{array}$ & $\begin{array}{l}3.1 \pm \\
0.06 \\
\end{array}$ & $\begin{array}{c}0.496 \\
* \mathrm{p}=.001\end{array}$ & $\begin{array}{c}3 \pm \\
0.01 \\
\end{array}$ & $\begin{array}{l}3.2 \pm \\
0.01 \\
\end{array}$ & $\begin{array}{c}0.46 \\
* \mathrm{p}=.004\end{array}$ \\
\hline
\end{tabular}

Legend to Table 5: GGT- $\gamma$ glutamyl trasnferase, AST- Aspartate transaminase, ALT- Alanine transaminase, TP- Total protein, Alb-Albumin, SE- Standard error. BW- Before withdrawal, AWAfter withdrawal, r-correlation coefficient. GGT, AST, ALT, and Glb show significant correlation between before and after withdrawal levels. TP was significant in alcohol dependent smokers in both before and after withdrawal. Alb was not significant in any groups. $\mathrm{p}<.05-$ significant.

\section{Discussion}

The significant decrease observed in our study in the levels of GGT, AST and ALT were not only in alcohol dependent non-smokers but also in alcohol dependent smokers. This finding was seen in blood as well as saliva. As observed in our study, several studies have corroborated our finding that even though smoking enhances the effect of alcohol it does not cause direct liver injury. ${ }^{17}$ Alcohol leads to inflammation with increase in the inflammatory liver enzymes GGT, AST and ALT levels as observed in our study. ${ }^{18}$ However after withdrawal we observed significant normalization in the liver enzymes, this could be due to the significant recovery from the alcohol induced hepatic inflammatory activity.

The normalization rates of liver enzymes in this study after deaddiction were very similar in blood as well as saliva which is concordant with other studies. ${ }^{19}$ Hence salivary liver function enzymes can be used to monitor the prognosis of the deaddiction treatment in both alcohol dependent smokers as well as non-smokers. However large population based studies are needed to validate this.

The recovery from the liver inflammation usually leads to improvement in synthetic functions of the hepatocytes. ${ }^{20}$ This is responsible for the elevated levels of the TP and Alb in saliva as well as blood as observed in our study but it was not significant. In our study the after withdrawal levels of total protein and albumin were measured varying from 19-21 days of after deaddiction, which is almost equal to the half life of albumin. ${ }^{21}$ This clearly indicates hepatocytes require longer time to regain their synthetic activity. The expected elevation in TP levels after withdrawal was not as anticipated because of the concomitant decrease in the globulin levels, which could be due to recovery from the alcohol induced hepato inflammatory activity. 
The decrease observed in Glb after withdrawal was significant only in blood of alcohol dependent non-smokers. The additive enhancing inflammatory effect of smoking is responsible for the persistent elevation of the globulins after withdrawal in alcohol dependent smokers (Table 2 and 3 ).

Total protein and albumin levels after withdrawal cannot be predicted using values of before withdrawal levels in blood as well as saliva in both alcohol dependent smokers and non-smokers. The increase in total protein and albumin observed after withdrawal is not uniform with respect to their before withdrawal levels (Table 4 and 5).

Further studies are needed to prove this hypothesis that hepato inflammatory activity recovers earlier than the regain in the hepatic synthetic activity after deaddiction. Serum and salivary liver enzymes should be correlated with hsCRP and other inflammatory markers.

\section{Conclusion}

In both alcohol dependent smokers as well as non-smokers, salivary GGT, AST and ALT levels can be used instead of blood levels to monitor the prognosis of deaddiction, but TP and Alb cannot be used for the same.

Acknowledgements: All the authors wish to thank the authority of Father Muller Medical College for providing support and infrastructure.

Funding: This study was not funded.

\section{Compliance with ethical standards}

Conflict of Interest: JB Honnamurthy, AR

Shivashankara, SS Avinash, P JohnMathai, and M Malathi declare that they have no conflict of interest.

\section{Human and Animal Rights Statement: All procedures performed in studies involving human participants were in accordance with the ethical standards of the institutional research committee and with the 1964 Helsinki declaration and its later amendments or comparable ethical standards.}

Ethical approval: The study was approved by Father Muller Institutional ethics committee. (FMMC/FMIEC/2039/2014).

Informed consent: All authors declare that written informed consent was obtained from all individual participants included in the study.

\section{References}

1. World Health Organisation. Global status report on alcohol and health 2014. Glob status Rep alcohol. 2014;1-392.

2. Jang ES, Jeong S-H, Hwang SH, Kim HY, Ahn SY, Lee J, et al. Effects of coffee, smoking, and alcohol on liver function tests: a comprehensive cross-sectional study. BMC Gastroenterol [Internet]. BMC Gastroenterology; 2012;12(1):145.

3. True WR, Xian H, Scherrer JF, Madden PAF, Bucholz KK, Heath AC, et al. Common Genetic Vulnerability for Nicotine and Alcohol Dependence in Men. 2016;56:655-61.

4. Treatment D. Changes of Serum Transaminases in Chronic Alcoholics Undergoing. 2015;3(3):4651-5.

5. Breitling LP1, Raum E, Müller H, Rothenbacher D BH. Synergism between smoking and alcohol consumption with respect to serum gammaglutamyltransferase. Hepatology. 2009;49(3):802-8.

6. Zhang C-Z, Cheng X-Q, Li J-Y, Zhang P, Yi P, $\mathrm{Xu} X$, et al. Saliva in the diagnosis of diseases. Int J Oral Sci [Internet]. Nature Publishing Group; 2016;8(3):133-7.

7. Bosch JA. The use of saliva markers in psychobiology: mechanisms and methods. Monogr Oral Sci. 2014;24:99-108.

8. Zhang Y, Sun J, Lin CC, Abemayor E, Wang MB WD. The emerging landscape of salivary diagnostics. Periodontol 2000. 2016;70(1):38-52.

9. World Health Organization. The ICD-10 classification of mental and behavioural disorders: clinical descriptions and diagnostic guidelines. WorldHealthOrganization 1992;1267.

10. Navazesh M. Methods for collecting saliva. Ann N Y Acad Sci. 1993;694:72-4.

11. Committee of enzymes of the Scandinavian society for clinical chemistry and clinical physiology. Recommended method for the determination of gamma-glutamyl transferase in blood. Scand J Clin Lab Invest. 1976;36:19-125.

12. Bergmeyer HU, Hørder M RR. International Federation of Clinical Chemistry (IFCC) Scientific Committee, Analytical Section: approved recommendation (1985) on IFCC methods for the measurement of catalytic concentration of enzymes. Part 2. IFCC method for aspartate aminotransferase. J Clin Chem Clin Biochem. 1986;24(7):497-510.

13. Bergmeyer HU, Hørder M RR. International Federation of Clinical Chemistry (IFCC) Scientific Committee, Analytical Section: approved recommendation (1985) on IFCC methods for the measurement of catalytic concentration of enzymes. Part 3. IFCC method for alanine aminotransferase. J Clin Chem Clin Biochem. 1986;24(7):481-95.

14. Doumas BT, Peters T. Serum and urine albumin: a progress report on their measurement and clinical significance. Clin Chim Acta . 1997;258(1):3-20.

15. Lowry OH, Rosebrough NJ, Farr AL RR. Protein measurement with the Folin phenol reagent. J Biol Chem. 1951;193(1):265-75. 
16. Gornall AG, Bardawill CJ, David MM.

Determination of serum proteins by means of the biuret reaction. J Biol Chem. 1949;177(2):751-

66.

17. Wannamethee SG, Shaper AG. Cigarette smoking and serum liver enzymes: the role of alcohol and inflammation. Ann Clin Biochem. 2010;47(Pt 4):321-6.

18. M. de la Monte S, M T. Tobacco Smoke-Induced Hepatic Injury with Steatosis, Inflammation, and Impairments in Insulin and Insulin-Like Growth Factor Signaling. J Clin Exp Pathol. 2016;6(2):110.

19. Shivashankara AR, Johnny C, Malathi M, Arun Kumar K, Avinash SS, Thomas T. A correlative study on the aminotransferases and gamma glutamyl transferase in the saliva and serum of chronic alcoholics before and after alcohol deaddiction. J Clin Diagnostic Res. 2011;5(3):512-5.

20. Duarte S, Baber J, Fujii T, Coito AJ. Matrix metalloproteinases in liver injury, repair and fibrosis. Matrix Biol [Internet]. Elsevier B.V.; 2015;44-46:147-56.

21. Bennhold H. Kallee E, . Comparative studies on the half-life of I 131-labeled albumins and nonradioactive human serum albumin in a case of analbuminemia. J Clin Invest. 1959;38(5):86372. 